\title{
Preeclampsia Is Associated with Failure of Human Cytotrophoblasts to Mimic a Vascular Adhesion Phenotype One Cause of Defective Endovascular Invasion in This Syndrome?
}

\author{
Yan Zhou, ${ }^{*}$ Caroline H. Damsky, ${ }^{\star *}$ and Susan J. Fisher ${ }^{\star} \neq \$ \|$ \\ *Department of Stomatology, ${ }^{\ddagger}$ Department of Obstetrics, Gynecology and Reproductive Sciences, ${ }^{\S}$ Department of Pharmaceutical \\ Chemistry, and ${ }^{\| D}$ epartment of Anatomy, University of California at San Francisco, San Francisco, California 94143
}

\begin{abstract}
In human pregnancy, placental cytotrophoblasts that invade the uterus downregulate the expression of adhesion receptors that are characteristic of their epithelial origin, and upregulate the expression of adhesion receptors that are expressed by vascular cells. We suggest that this transformation could be critical to endovascular invasion, the process whereby cytotrophoblasts invade the uterine spiral arterioles and line their walls (Zhou et al. J. Clin. Invest. 1997. 99: 2139-2151.). To better understand the in vivo significance of these findings, we tested the hypothesis that in preeclampsia, an important disease of pregnancy in which endovascular invasion is abrogated, cytotrophoblasts fail to adopt a vascular adhesion phenotype. In experiments described here we stained placental bed biopsy specimens from age-matched control pregnancies and from those complicated by preeclampsia with antibodies that recognize adhesion molecules that are normally modulated during this transformation. In preeclampsia, differentiating/invading cytotrophoblasts fail to express properly many of these molecules, including integrin, cadherin, and Ig superfamily members. These results suggest that preeclampsia is associated with failure of cytotrophoblasts to mimic a vascular adhesion phenotype. The functional consequences of this abnormality are unknown, but are likely to affect negatively cytotrophoblast endovascular invasion and uterine arteriole remodeling, thereby compromising blood flow to the maternal-fetal interface. (J. Clin. Invest. 1997. 99:2152-2164.) Key words: placenta $\bullet$ endothelium • vascular $\bullet$ uterus • cadherins • integrins
\end{abstract}

\section{Introduction}

Preeclampsia is a disease that adversely affects $7-10 \%$ of first pregnancies in the United States (1). The mother shows signs and symptoms that suggest widespread alterations in endothelial function (e.g., high blood pressure, proteinuria, and edema [2]). In some cases the fetus stops growing, which leads to in-

Address correspondence to Susan J. Fisher, HSW 604, University of California, San Francisco, CA 94143-0512. Phone: 415-476-5297; FAX: 415-476-4204; E-mail: sfisher@cgl.ucsf.edu

Received for publication 18 September 1996 and accepted in revised form 27 January 1997.

J. Clin. Invest.

(C) The American Society for Clinical Investigation, Inc.

0021-9738/97/05/2152/13 \$2.00

Volume 99, Number 9, May 1997, 2152-2164 trauterine growth retardation. The severity of the disease varies greatly. In its mildest form the signs/symptoms appear near term and resolve after birth, with no lasting effects on either the mother or the child. In its severest form, the signs and symptoms occur in the second trimester. If they cannot be controlled, the only option is delivery, with consequent iatrogenic fetal prematurity. As a result of the latter form of the disease, preeclampsia (and hypertensive diseases of pregnancy) are a leading cause of maternal death and contribute significantly to premature deliveries in the United States (3).

Although the cause(s) of preeclampsia is unknown, compelling evidence implicates the placenta (4). The disease can occur in hydatidiform mole (i.e., in the absence of a fetus [5]), and removing the placenta (i.e., birth) cures preeclampsia. Histologic studies suggest that the part of the placenta that is most severely affected is the portion which attaches to the uterine wall (6-9). Detailed analyses of this area (e.g., placental bed biopsies) show that in preeclampsia, as compared with normal pregnancy, cytotrophoblast invasion is shallow. Although the depth of interstitial invasion varies, endovascular invasion does not proceed beyond the superficial portions of the uterine spiral arterioles. Thus, the histologic appearance of these vessels does not change: they retain their endothelial linings and muscular walls. Their physiological properties do not change either: spiral arterioles remain relatively narrow-bore, high-resistance vessels. This is in dramatic contrast to normal pregnancy, where cytotrophoblasts penetrate as far as the myometrial segments of spiral arterioles, thereby increasing by severalfold the diameter of these vessels. Hence, in preeclampsia flow of maternal blood to the feto-placental unit is significantly reduced relative to normal pregnancy. Failure of these changes to occur is highly relevant to the disease process, since reduced placental perfusion is an important risk factor for developing preeclampsia (10).

We hypothesized that these anatomical defects were important clues for uncovering the underlying molecular defects. Data in support of this hypothesis come from our studies of cytotrophoblast differentiation along the invasive pathway in normal pregnancy and in preeclampsia. In normal pregnancy, cells that are committed to differentiate along this pathway upregulate the expression of molecules that play a role in invasion (e.g., integrin $\alpha 1[11,12]$ and matrix metalloproteinase-9 [13]) and in modulating the maternal immune response (HLA-G $[14,15])$. In preeclampsia, analogous cells (hereafter referred to as preeclamptic cytotrophoblasts) fail to express normal levels of these stage-specific antigens, suggesting that their ability to differentiate/invade is impaired (16-17, Lim, K.H., Y. Zhou, M. Janatpour, M. McMaster, K. Bass, S.-H. Chun, and S.J. Fisher, manuscript submitted for publication).

Likewise, we hypothesized that the failure of preeclamptic cytotrophoblasts to invade the spiral arterioles pointed to the 
importance of this process in normal pregnancy. Although many cells can adopt an invasive phenotype (e.g., tumor cells) or transiently adhere to endothelium (e.g., leukocyte tethering), formation of stable associations with blood vessels appears to be a unique property of cytotrophoblasts. We further hypothesized that this unusual behavior would require an equally unusual mechanism, namely that in normal pregnancy, those cytotrophoblasts that remodel the spiral arterioles might broadly mimic the adhesion phenotype of the vascular cells they replace. Experiments described in the accompanying paper (18) confirmed this hypothesis. Here we show that preeclamptic cytotrophoblasts do not stain for most of the endothelialtype adhesion molecules that are expressed by control cytotrophoblasts, suggesting that they fail to undergo this important phenotypic transformation. We suspect that this failure plays an important role in the pathophysiology of the disease.

\section{Methods}

Placental sources. Women who received prenatal care at the University of California San Francisco (Moffitt/Long Hospital, San Francisco General Hospital) and who had no underlying medical conditions were enrolled in this study. During one of the late second trimester/ early third trimester clinic visits, informed consent was obtained to remove a placental bed biopsy specimen if a cesarean section was performed. The consent form and procedure for performing the biopsy were approved by the University of California San Francisco Committee on Human Research.

Samples of floating chorionic villi, basal plate, and the placental bed, all from the same pregnancy, were obtained during cesarean section from both control patients and those with preeclampsia. Placental bed specimens were collected by direct visualization of the placental site. Patients with preeclampsia were diagnosed according to the following criteria, recommended by Chesley (19): nulliparity; no history of hypertension prior to pregnancy; increase in diastolic pressure of $15 \mathrm{mmHg}$ or systolic pressure of $30 \mathrm{mmHg}$ compared with blood pressure obtained before $20 \mathrm{wk}$ of gestation; proteinuria $\geq 0.5 \mathrm{~g} / 24 \mathrm{~h}$ or $\geq 30 \mathrm{mg} / \mathrm{dl}$ (or $1+$ on urine dipstick) in a catheterized specimen; hyperuricemia $>5.5 \mathrm{mg} / \mathrm{dl}$ (or one SD greater than the normal mean value prior to term); return to normal blood pressure, and resolution of proteinuria by $12 \mathrm{wk}$ postpartum. The American College of Obstetricians and Gynecologists' definitions for severe preeclampsia (20) and published criteria for the syndrome of hemolysis, elevated liver enzymes, and low platelets (HELLP) ${ }^{1}$ were used (21): systolic blood pressure of $\geq 160 \mathrm{mmHg}$ and/or diastolic pressure of $\geq 110 \mathrm{mmHg}$; proteinuria of $\geq 5 \mathrm{gm}$ in a 24 -h period or $3+$ on urine dipstick; presence of cerebral or visual disturbances; epigastric pain; oliguria $(<500 \mathrm{ml}$ in $24 \mathrm{~h}$ ); pulmonary edema; evidence of hemolysis on peripheral smear; increased bilirubin $\geq 1.2 \mathrm{mg} / \mathrm{dl}$; increased lactic dehydrogenase $>600$ IU/liter; platelet counts $<100,000 / \mathrm{mm}^{3}$; serum glutamic-oxaloacetic transaminase level of $\geq 70 \mathrm{IU} / \mathrm{ml}$. Eclampsia was defined as an onset of seizure activity in the setting of preeclampsia in a patient without prior history of seizure disorder. 10 samples were obtained from preeclamptic patients at 26-35 wk of gestation. Eight were diagnosed with severe preeclampsia (two at $26 \mathrm{wk}$; one each at 27, 28, 29, 30, 33, and $35 \mathrm{wk}$ of gestation), one with HELLP syndrome (26 wk), and one with eclampsia/severe preeclampsia (26 wk).

None of the control patients had evidence of preeclampsia, gestational hypertension, chorioamnionitis, chronic hypertension, or a medical history that suggested they were at an increased risk for develop-

1. Abbreviations used in this paper: HELLP, the syndrome of hemolysis, elevated liver enzymes, and low platelets; PECAM-1, platelet endothelial adhesion molecule-1; VCAM-1, vascular adhesion molecule-1. ing preeclampsia. None of the placentas had abnormalities that could be detected either grossly or histologically. Eight control samples were obtained from women who were between 22 and $36 \mathrm{wk}$ gestation. Of these, one 22-wk sample was taken from a nulliparous woman who had a previous history of cervical cancer, whose disease recurred locally during pregnancy and who elected to have a late second trimester hysterectomy. Two other samples were obtained from women who were delivered at $26 \mathrm{wk}$ : one because of cervical incompetence, and the other because of nonoperable, conjoined twins. Five placental bed specimens were obtained from control, nulliparous women who underwent cesarean sections at 33-36 wk for obstetrical indications other than preeclampsia.

Antibodies, tissue fixation, and immunocytochemistry. With the exceptions noted, antibodies, tissue preparation and specific methods were described in (18). For localization of cytotrophoblasts by using light microscopy, sections were incubated with a primary anti-cytokeratin antibody overnight at $4^{\circ} \mathrm{C}$, and then with secondary antiserum, peroxidase-conjugated goat anti-rat IgG (Jackson ImmunoResearch Labs, Inc., West Grove, PA; 1:200), for $45 \mathrm{~min}$ at room temperature. The peroxidase reaction was developed using 3,3'-diamino-benzidine and the sample was counterstained with Gill's hematoxylin. Endothelial cells were localized by using a mouse monoclonal antibody (F8/ 86) that recognized human vWf, which was purchased from DAKO A/S, Glostrup, Denmark.

\section{Results}

Interactions between invasive (fetal) cytotrophoblasts and uterine (maternal) blood vessels in control pregnancies. Important steps in cytotrophoblast invasion of uterine arterioles are depicted in Fig. 1. To differentiate cytotrophoblasts, these tissue sections were stained with anti-cytokeratin, then peroxidaseconjugated secondary antibodies. In the first step, cytotrophoblasts form cell columns that act as a bridge between the anchoring villi and the uterine wall (Fig. $1 A$ ). Once they are inside the uterine wall, some of the cytotrophoblasts invade the parenchyma. Others target uterine vessels; the cells extensively invade arteries, but not veins (Fig. $1 B$ ). Large-bore arterioles contain plugs of cytotrophoblasts. The fetal cells replace the endothelial lining and invade the tunica media of these maternal vessels (Fig. $1 C)(8,9)$.

Interactions between invasive (fetal) cytotrophoblasts and spiral (maternal) arterioles are altered in preeclampsia. The effect of preeclampsia on endovascular invasion is particularly evident when interactions between fetal cytotrophoblasts and maternal endothelial cells are studied in detail. For this purpose we double stained sections with an antibody that recognizes cytokeratin, and thereby identifies cytotrophoblasts, and with an antibody that reacts with vWf, and thereby identifies endothelial cells. In control pregnancy, cytotrophoblasts have two types of interactions with maternal arterioles (Fig. 2, $A-D$ ). In the first, large aggregates of these fetal cells are found primarily inside the vessel lumen (Fig. $2 A$ ). These aggregates can either lie adjacent to the apical surface of the resident endothelium (as marked by the asterisk in the left side of the vessel in Fig. 2, $A$ and $B$ ) or replace it such that they appear directly attached to the vessel wall (as marked by the white arrowhead in the right side of this vessel). In the second type of interaction, cytotrophoblasts are found within the vessel wall (Fig. $2 C$ ) rather than in the lumen. In this position they colonize the smooth muscle layer of the vessel and lie subjacent to the endothelium (Fig. $2 D$ ). These different types of interactions may be progressive stages in a single process, or indicative of different strategies by which cytotrophoblasts accomplish endovas- 

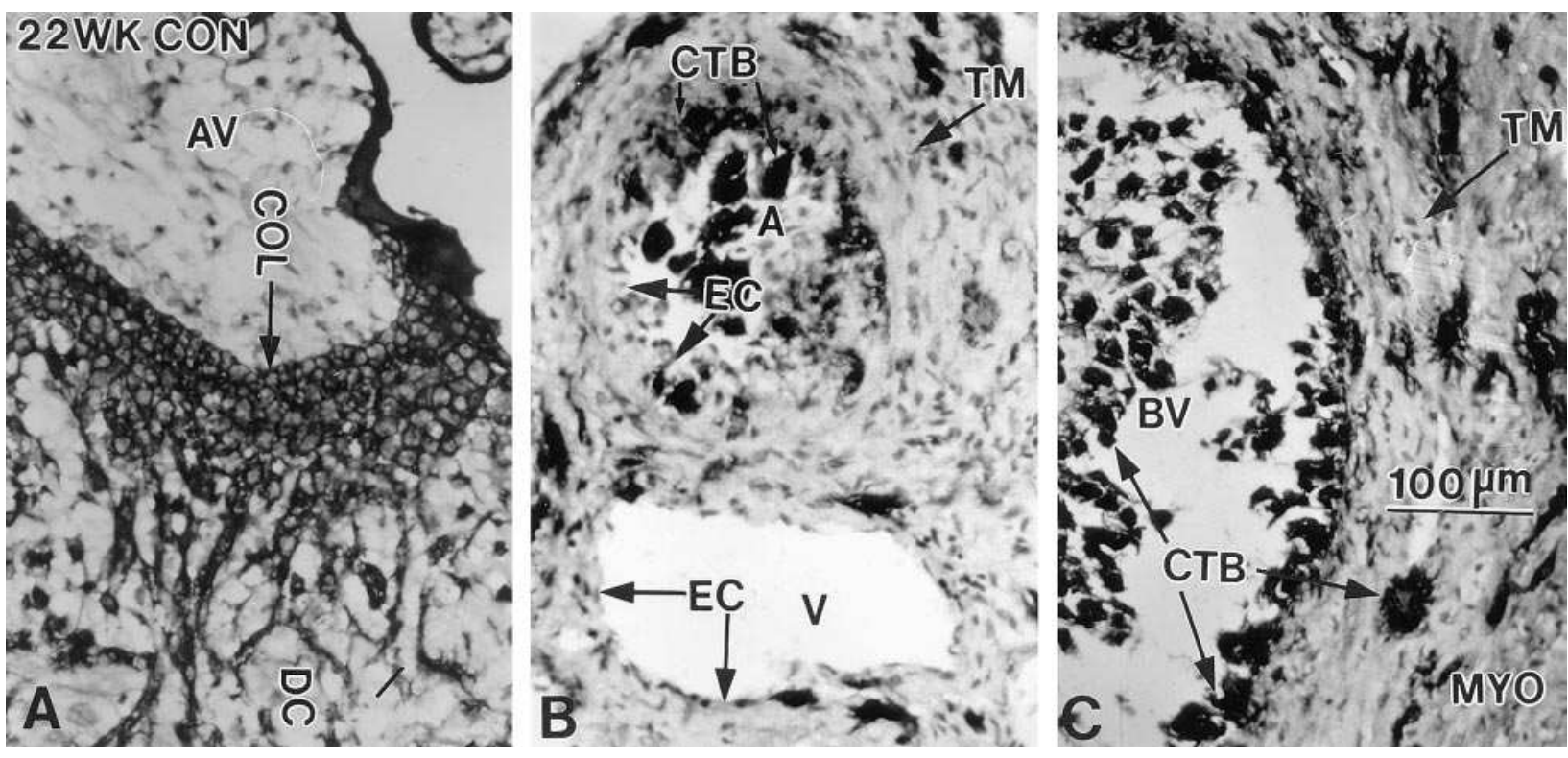

Figure 1. Cytotrophoblast interactions with the uterus during the second trimester of control pregnancy. The sections were stained with anti-cytokeratin, then peroxidase-conjugated secondary antibodies to identify cytotrophoblasts. $(A)$ Section of the maternal-fetal interface where a cytotrophoblast column $(C O L)$ attaches an anchoring villus $(A V)$ to the decidua $(D C)$. $(B)$ Section of the uterine wall that shows cytotrophoblast $(C T B)$ interactions with maternal vessels that are still lined by endothelial cells $(E C)$. Note that fewer cytotrophoblasts associate with the vein $(V)$ than with the artery $(A)$, whose tunica media $(T M)$ is still intact. $(C)$ Section of the myometrial portion of a uterine arteriole that has been extensively invaded by cytotrophoblasts. Fetal cells are found in the vessel lumen, along the wall and within the tunica media.

cular invasion. In either case, the stage in which fetal cytotrophoblasts cohabit with maternal endothelium in the spiral arterioles is transient. By late second trimester, these vessels are lined exclusively by cytotrophoblasts (9); endothelial cells are no longer visible in either the endometrial or the superficial portions of their myometrial segments (data not shown).

In preeclampsia, cytotrophoblast interactions with the spiral arterioles are very different. Serial sections through placental bed biopsies of all the patients we studied showed that few of the spiral arterioles contained cytotrophoblasts. Instead, most cytotrophoblasts remained at some distance from these vessels (Fig. 2, $E$ and $F$ ). In locations where endovascular cytotrophoblasts were detected, invasion was limited to the portion of the vessel that spans the superficial decidua. Thus, there was little difference between their interactions with uterine veins and arterioles. Even if the cytotrophoblasts gained access to the lumen, they usually failed to form tight aggregates among themselves, or to spread out on the vessel wall, as was observed for cytotrophoblasts in control samples matched for gestational age. Instead, preeclamptic cytotrophoblasts that reached the uterine arterioles tended to remain as individual rounded cells, suggesting that they were poorly anchored to the vessel wall (e.g., Fig. 3, $K$ and $L$; see also Fig. 6, $G$ and $H$ ). Thus, cytotrophoblasts in preeclampsia not only have a limited capacity for endovascular invasion $(6,9)$ but also display an altered morphology in their interactions with maternal arterioles.

The hypotheses tested in this paper were based on these morphological observations. We also considered the results of other relevant studies from our laboratories. First, we find that in preeclampsia, column cytotrophoblasts and cytotrophoblasts in the decidua fail to downregulate integrin $\alpha 6 \beta 4$ and to upregulate integrin $\alpha 1$ expression (9), an important feature of normal placentation (11). Second, we show that during normal placentation, cytotrophoblasts differentiating along the invasive pathway downregulate adhesion receptors that are characteristic of their epithelial origin and switch to an adhesion phenotype that broadly mimics that of vascular cells (18). Together, these results suggested the possibility that preeclampsia negatively affects the ability of cytotrophoblasts to express adhesion molecules that are normally modulated during this unique epithelial-to-vascular transformation.

In preeclampsia, cytotrophoblasts differentiating along the invasive pathway fail to correctly modulate their expression of $\alpha V$-family integrins. We compared cytotrophoblast expression of three members of the $\alpha \mathrm{V}$-family $(\alpha \mathrm{V} \beta 5, \alpha \mathrm{V} \beta 6$, and $\alpha \mathrm{V} \beta 3)$ in placental bed biopsies obtained from control and preeclamptic patients that were matched for gestational age. In control samples, anti- $\beta 5$ staining during the latter half of the second trimester was very weak and was limited to cytotrophoblast stem cells in a subset of floating and anchoring villi. Cytotrophoblasts within the basal plate did not stain for this receptor (data not shown). The pattern was similar to that observed earlier in gestation (18), although staining became progressively weaker with each successive trimester (data not shown).

At $26 \mathrm{wk}$ staining for the $\beta 6$ subunit in control samples was largely restricted to villus cytotrophoblast stem cells at sites of column initiation. Antibody reactivity was detected only on cytotrophoblasts that were in contact with the trophoblast basement membrane, and did not extend to cells within the columns (Fig. 3 B). This pattern was similar to, but even more restricted than, that found in the normal placental bed earlier in gestation. In the latter case $\beta 6$ staining extended to the initial 1-2 cell layers of the cell column (18). Staining for $\beta 3$ at 26 wk of gestation was absent on villus and proximal column 

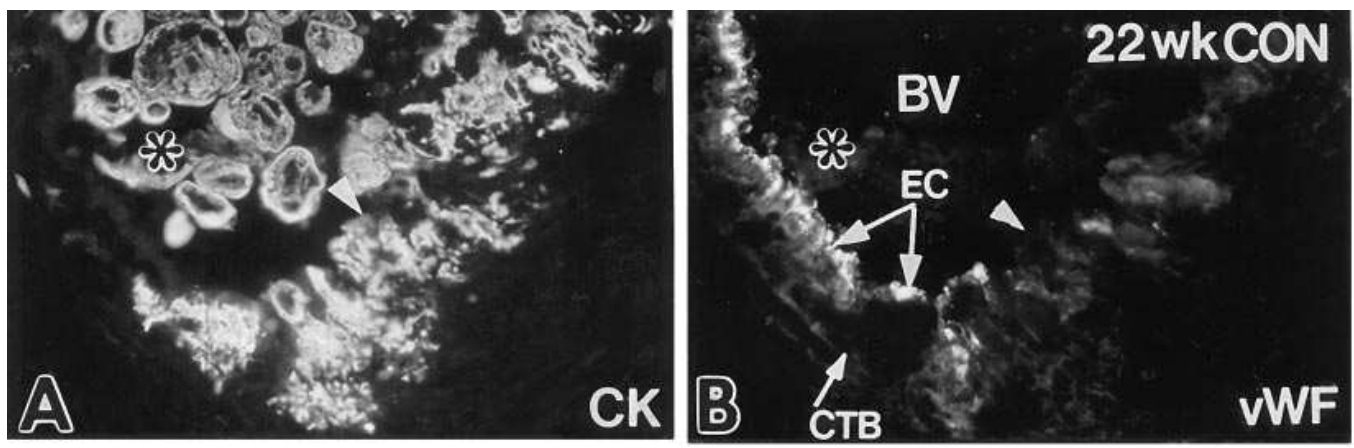

Figure 2. Cytotrophoblast interactions with endothelial cells were changed in preeclampsia. $(A-D)$ Sections of maternal spiral arterioles in control second trimester placental bed biopsy tissue at sites where cytotrophoblasts $(C T B)$ are replacing endothelial cells $(E C) .(E, F)$ Section of maternal spiral arteriole in 26 wk placental bed biopsy tissue from patient with severe preeclampsia $(S P E)$. Panels show the relationship of CTB, stained by anti-cytokeratin ( $C K, A, C$, and $E$ ), and maternal EC, stained with anti-vWf $(B$, $D$, and $F)$. $(A, B)$ Cross section of a blood vessel $(B V)$ in which CTB have several distinct interactions with EC. In one area (*), a large aggregate of CTB within the vessel lumen appears to contact the endothelial layer. In another area, CTB have replaced the EC lining (white arrowhead). At the junction of the two areas CTB lie deep to the remaining EC (arrows). ( $C$, $D)$ A patch of cytotrophoblasts in the vessel wall deep to endothelial cells. At the site of the black arrowhead, the EC appear to be sloughing off, leaving

behind the well-anchored CTB. $(E, F) \mathrm{BV}$ within a preeclamptic placental bed. Note that the vessel diameter is considerably smaller than those in control tissue. The vessel wall is lined completely by an intact EC layer and is surrounded by a thick tunica media (TM). CTB are in the surrounding uterine wall interstitium at a distance from the BV. No CTB are within the BV lumen.

cytotrophoblasts. But strong staining was detected on cytotrophoblasts in the distal column, the uterine wall (Fig. $3 F$ ) and maternal vessels (Fig. $3 \mathrm{~J}$ ). This pattern was similar to that found in tissue from earlier gestational ages, and did not substantially change during the third trimester.

We found that preeclampsia changed cytotrophoblast expression of all three $\alpha \mathrm{V}$-family members. When samples were matched for gestational age, even fewer preeclamptic cytotrophoblast stem cells stained with an antibody that recognized integrin $\beta 5$ (data not shown). In contrast, staining for $\beta 6$ was much brighter in preeclamptic tissue and extended beyond the column to include cytotrophoblasts within the superficial decidua (Fig. $3 D$ ). Interestingly, staining for $\beta 3$ was weak on cytotrophoblasts in all locations; cytotrophoblasts in the uterine wall of preeclamptic patients failed to show strong staining for $\beta 3$ (Fig. $3 H$ ), as did cytotrophoblasts that penetrated the spiral arterioles (Fig. $3 \mathrm{~L}$ ). Thus, in preeclampsia differentiating/invading cytotrophoblasts retain expression of $\alpha \mathrm{V} \beta 6$, which is transiently expressed in remodeling epithelium (22), and fail to upregulate $\alpha \mathrm{V} \beta 3$, which is characteristic of angiogenic endo- thelium (23). Therefore, as was the case for integrin $\alpha 1$ (9), our analyses of the expression of $\alpha \mathrm{V}$-family members suggests that in preeclampsia, cytotrophoblasts start to differentiate along the invasive pathway but cannot complete this process.

In preeclampsia, cytotrophoblasts differentiating along the invasive pathway fail to switch cadherin expression. We also examined cytotrophoblast expression of cadherins, molecules that mediate $\mathrm{Ca}^{2+}$-dependent cell-cell adhesion. In control samples at 26 wk of gestation, E-cadherin staining was strong on villus cytotrophoblasts, but was not detected on endothelial cells in either the placenta proper or the uterus. Expression of this adhesion molecule was greatly reduced on cytotrophoblasts in the superficial decidua, but was upregulated once the cells penetrated more deeply (Fig. 4 B). Cytotrophoblasts that invaded maternal spiral arterioles showed little or no staining for E-cadherin (see Fig. $4 \mathrm{D}$ ). This pattern was similar to that observed in control samples obtained at 10 and 22 wk of gestation (18). However, in normal term placental tissue, staining for E-cadherin was bright on cytotrophoblasts in all locations (data not shown). 

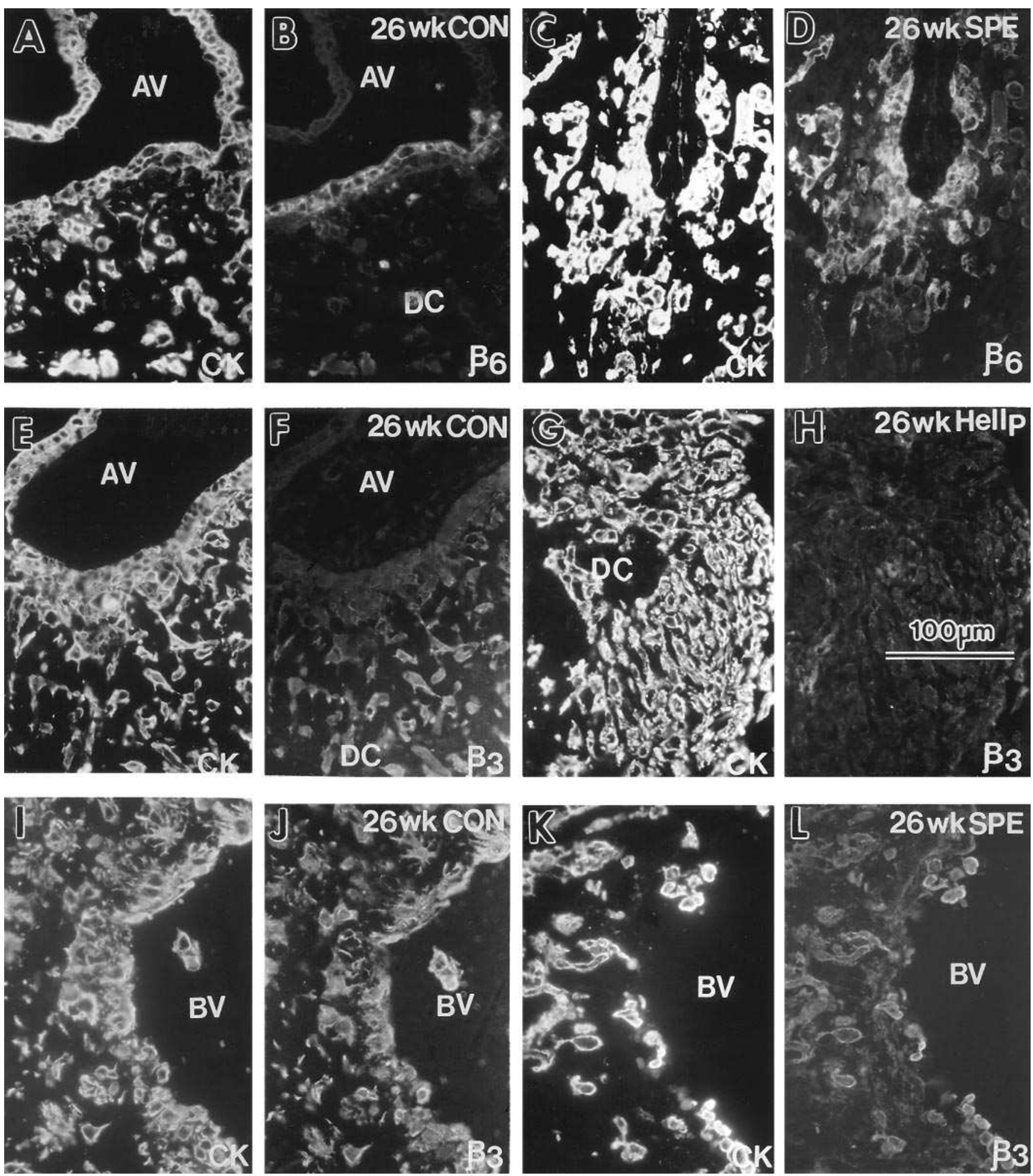

Figure 3. $\alpha \mathrm{V}$-family integrins were misexpressed in preeclamptic CTB. Sections of age-matched 26-wk control $(C O N, A, B, E, F, I$, and $J)$, severe preeclamptic (SPE, $C, D, K$, and $L$ ) and syndrome of hemolysis, elevated liver enzymes and low platelets $(H e l l p, G$ and $H)$ placental tissue containing anchoring villi (AV) and decidua $(D C)$ with $\mathrm{CTB}$ are shown. Sections were double stained with anti-CK $(A, C, E, G, I$, and $K)$ and either anti- $\beta 6$ integrin $(B$ and $D)$ or anti- $\beta 3$ integrin $(F, H, J$, and $L)$. In SPE tissue, staining for $\beta 6$ extended into the decidua, whereas staining in control tissue was restricted to sites of column initiation. $\beta 3$ staining was strong in control tissue on CTB that had invaded the decidua $(F)$ and either very weak or not detected in preeclamptic tissue $(H)$. Likewise, anti- $\beta 3$ stained strongly CTB in BV of control tissue $(J)$, but staining was reduced on most of the CTB that were associated with the BV of an SPE patient $(L)$. In the latter sample, CTB that lined the vessel wall stained the brightest. Note the rounded appearance of the latter cells $(L)$ as compared to CTB that line a BV in control tissue $(J)$. 

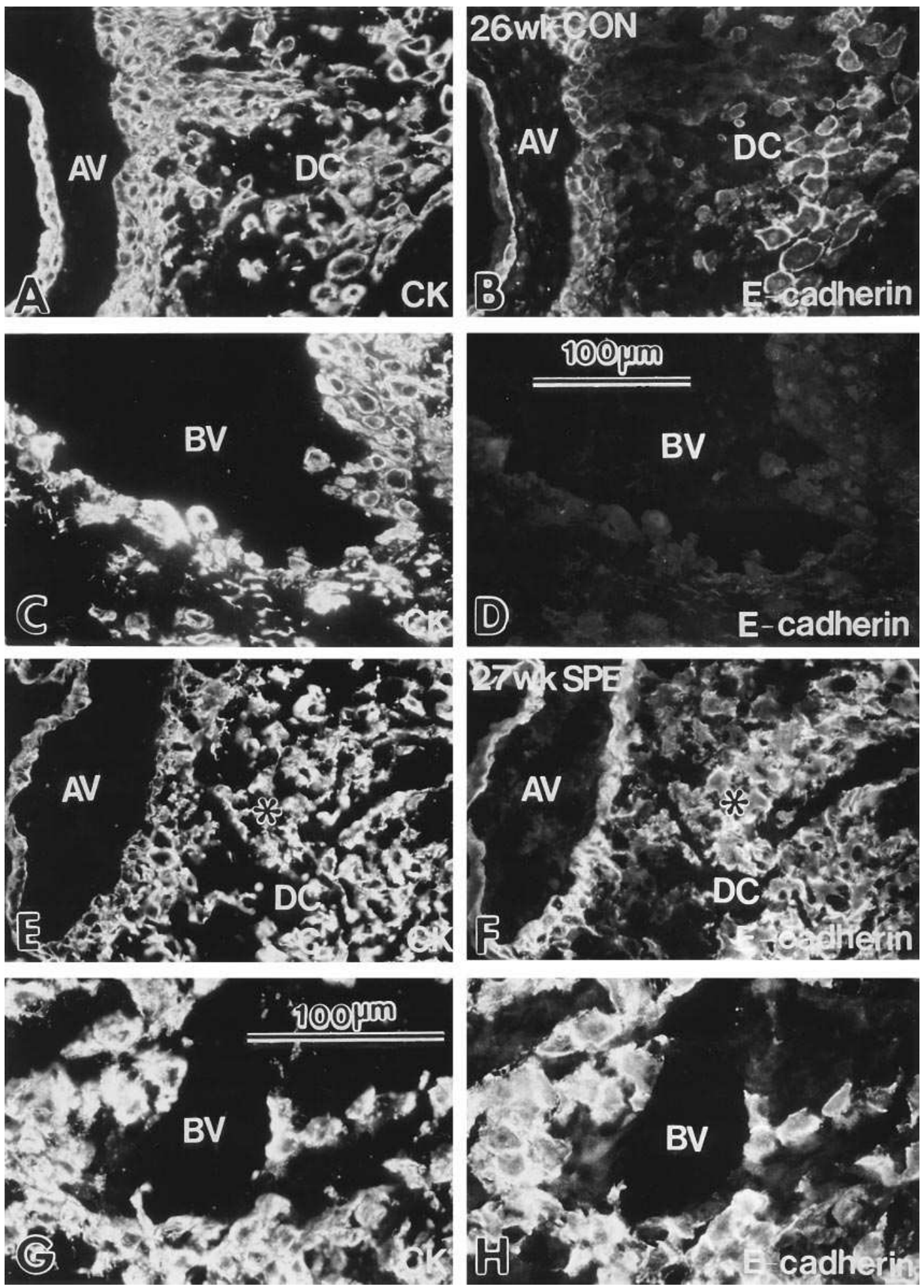
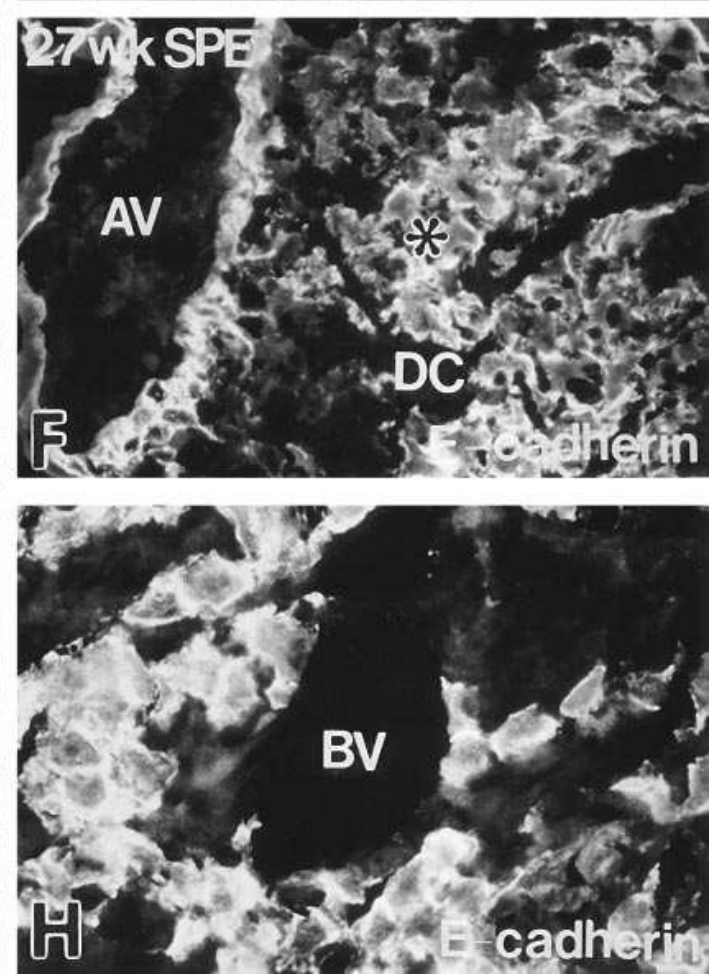

Figure 4. E-cadherin staining was transiently downregulated on CTB in control, but not in preeclamptic, placental tissue. Sections of 26-wk control $(C O N, A-D)$ and 27-wk severe preeclamptic ( $S P E, E-H)$ tissue double stained for CK $(A, C, E$, and $G)$ and E-cadherin $(B, D$, $F$, and $H) .(A, B)$ Control anchoring villus and placental bed. E-cadherin staining was strong on villus $\mathrm{CTB}$ and on CTB that invaded deeply into the uterine wall. Staining was reduced on CTB in cell columns and decidua. $(C, D)$ Section of myometrium containing a large maternal arteriole lined by CK-positive CTB. E-cadherin staining was not detected on BV-associated CTB. $(E, F)$ Section of preeclamptic anchoring villus and placental bed. E-cadherin staining was strong on CTB in almost all locations. CTB also appeared to be in large aggregates $(*)$. $(G, H)$ Section of preeclamptic placental bed tissue showing a blood vessel and surrounding decidua. Aggregates of E-cadherin-positive CTB were also associated with the vessel.
Preeclampsia had a striking effect on cytotrophoblast expression of E-cadherin. Cytotrophoblasts in both the villi and decidua showed strong reactivity with anti-E-cadherin (Fig. $4 F$ ), and staining remained strong even on cytotrophoblasts that had penetrated the superficial portions of uterine arterioles (Fig. $4 \mathrm{H}$ ). Interestingly, in preeclampsia we consistently found that cytotrophoblasts within the uterine wall tended to exist as large aggregates, rather than as smaller clusters and single cells, as is the case in normal pregnancy. This observation is in accord with the likelihood that E-cadherin mediates strong intercellular adhesion between cytotrophoblasts, as it does in all other normal epithelia examined (24).

To study cadherin modulation, we stained control and preeclamptic placental bed specimens that were matched for gestational age with anti-P-cadherin and anti-VE-cadherin. In control samples, we failed to detect cytotrophoblast P-cadherin 

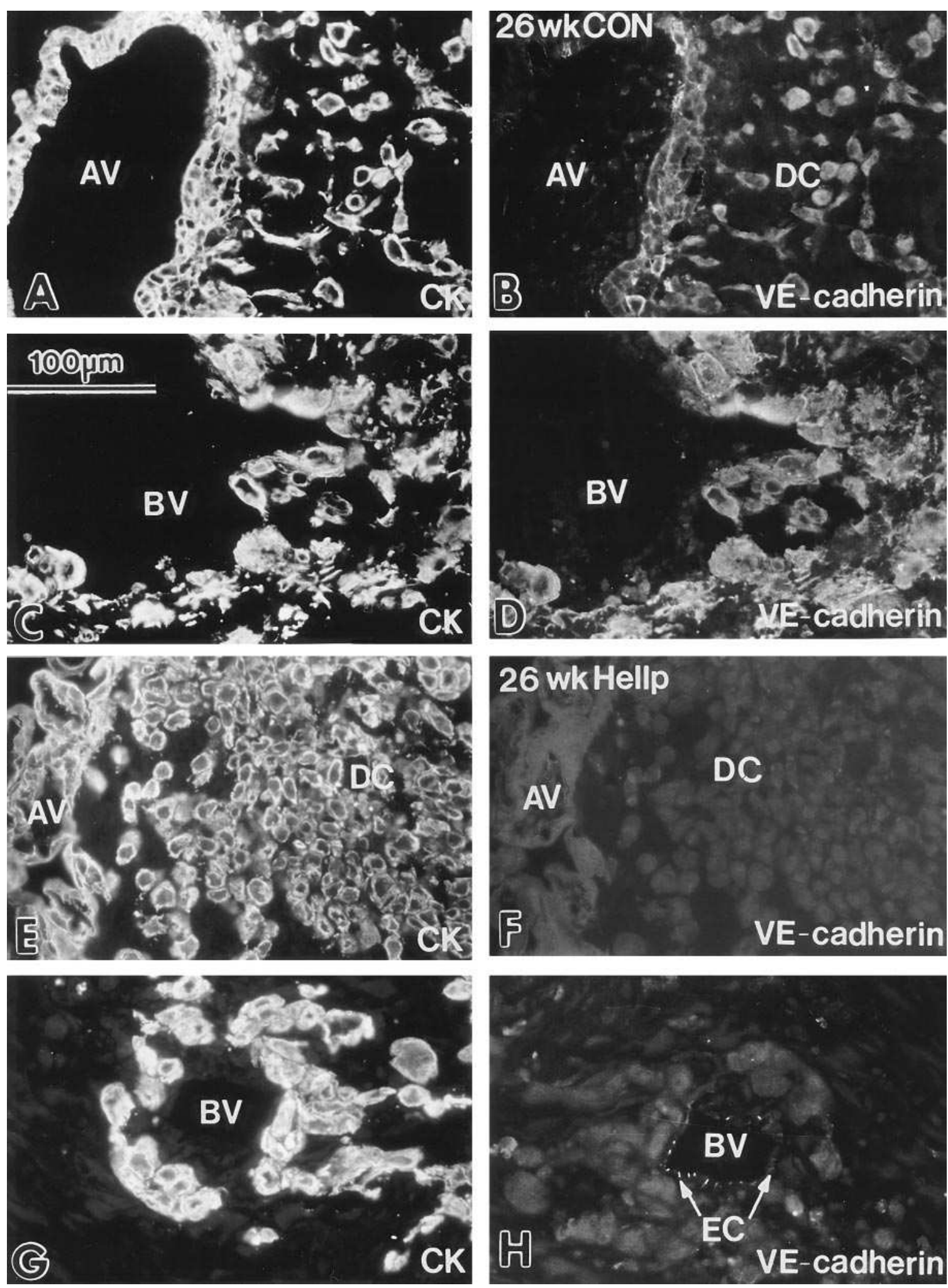

Figure 5. VE-cadherin was not detected on CTB in preeclamptic tissue. Sections of age-matched 26-wk control $(C O N, A-D)$ and HELLP preeclamptic $(P E, E-H)$ tissue double stained for CK $(A, C, E$, and $G)$ and VE-cadherin $(B, D, F$, and $H)$. $(A, B)$ Control anchoring villus and placental bed. VE-cadherin staining was not detected on villus CTB but was present on CTB within columns and the uterine wall. $(C, D)$ Section of a maternal spiral artery in control placental bed tissue. VE-cadherin staining was especially strong on intravascular CTB and CTB that were associated with the vessel wall. $(E, F)$ Section of preeclamptic anchoring villus and placental bed. VE-cadherin staining was not detected. $(G, H)$ Section through a small blood vessel surrounded by CTB. VE-cadherin was not detected on vessel-associated CTB or CTB within the surrounding tissue. However, the EC that line the vessel did stain (arrows). 

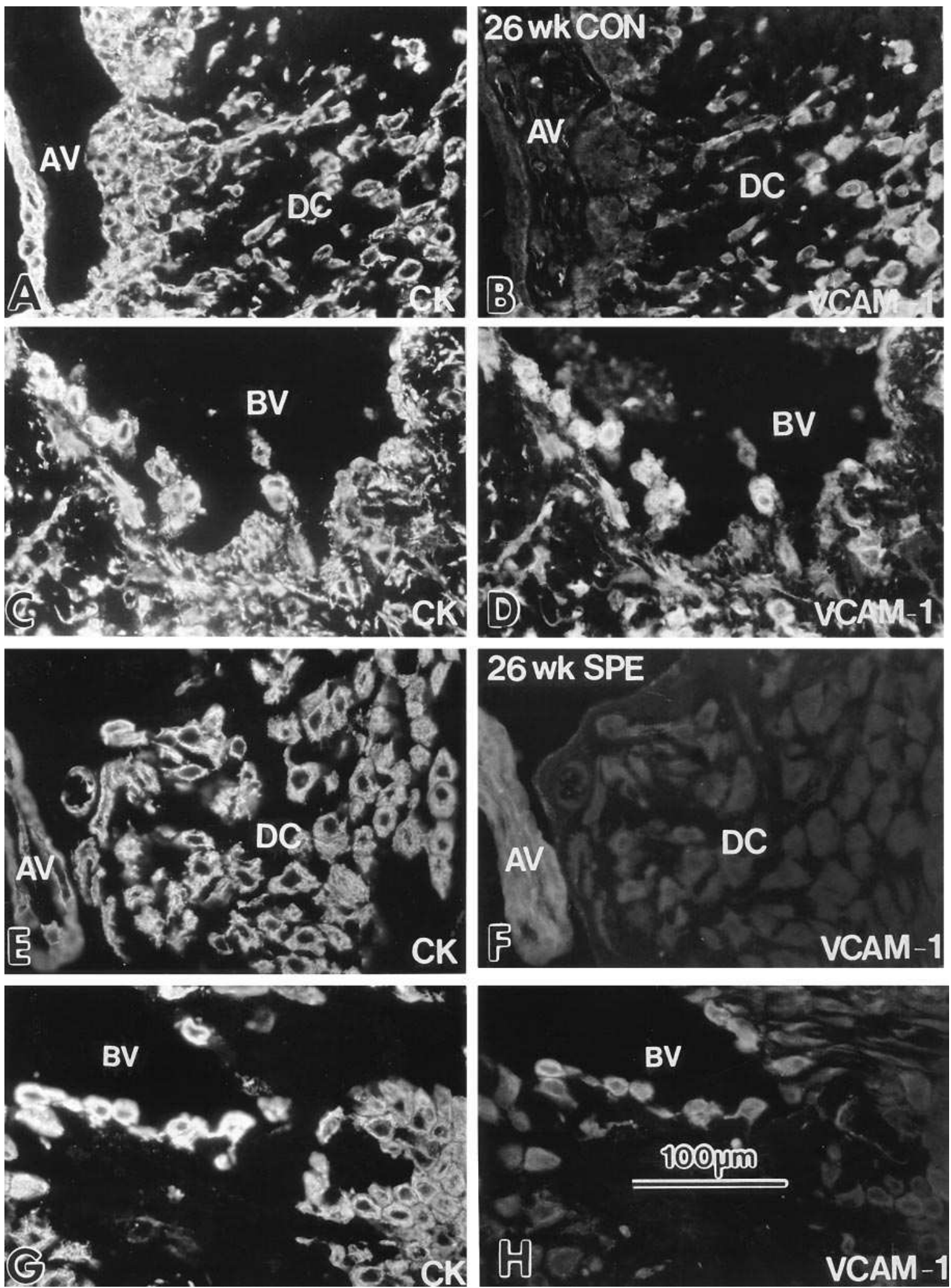

Figure 6. Staining for VCAM-1 was reduced in preeclamptic CTB. Sections of age-matched 26-wk control $(C O N, A-D)$ and severe preeclamptic $(S P E, E-H)$ tissue double stained for CK $(A, C, E$, and $G)$ and VCAM-1 $(B, D, F$, and $H)$, the counter receptor for $\alpha 4$ integrins. $(A, B) C o n t r o l$ anchoring villus and placental bed. Staining for VCAM-1 was not detected on villus CTB, but was increased on column and decidual CTB. $(C$, $D$ ) Maternal blood vessel lined with CTB. Staining for VCAM-1 was detected on both CTB associated with the blood vessel and on CTB in the surrounding uterine interstitium. In preeclamptic tissue, VCAM-1 was not detected on CTB in the uterine interstitium $(E, F)$, but weak staining for VCAM-1 was detected on CTB along the wall of a blood vessel $(G, H)$. 
expression during the second and third trimesters (data not shown). This was not unexpected since we also failed to detect its expression earlier in gestation (18). In contrast, at $26 \mathrm{wk}$ of a control pregnancy, both column and placental bed cytotrophoblasts stained for VE-cadherin (Fig. $5 \mathrm{~B}$ ). Staining for this adhesion molecule was particularly strong on endovascular cytotrophoblasts (Fig. $5 \mathrm{D}$ ). This pattern of VE-cadherin expression was essentially the same as that detected during the first trimester of pregnancy (18), and did not change during the third trimester (data not shown). However, the strongest staining was detected in the second trimester.

Strikingly, no VE-cadherin staining was detected on cytotrophoblasts in any location in placental bed specimens obtained from preeclamptic patients; neither cytotrophoblasts in the cell columns (Fig. $5 F$ ) nor the few cells that were found in association with vessels in the superficial decidua (Fig. $5 \mathrm{H}$ ) expressed VE-cadherin. However, staining for this adhesion molecule was detected on maternal endothelium in the unmodified uterine vessels in preeclamptic placental bed biopsy specimens (Fig. $5 H$ ). Thus, cadherin modulation by cytotrophoblasts in preeclampsia was defective, as shown by the persistence of strong E-cadherin staining and the absence of VE-cadherin staining on cytotrophoblasts in columns and in the superficial decidua.

In preeclampsia, cytotrophoblasts differentiating along the invasive pathway fail to upregulate expression of immunoglobulin superfamily adhesion receptors characteristic of endothelial cells. Vascular endothelial cells express several members of the Ig superfamily of adhesion receptors, many of which interact with leukocytes (25). In control pregnancy at 26 wk of gestation, vascular adhesion molecule-1 (VCAM-1), which interacts with integrins $\alpha 4 \beta 1$ and $\alpha 4 \beta 7$, is not detected on villus cytotrophoblasts, but is detected on cytotrophoblasts within the uterine wall (Fig. $6 \mathrm{~B}$ ). Staining is particularly strong on endovascular cytotrophoblasts (Fig. $6 \mathrm{D}$ ).

To determine whether the integrin receptors for VCAM-1 were expressed, these tissues were also stained with an antibody against the integrin $\alpha 4$ subunit. In control 26-wk samples, cytotrophoblasts in the villi and in the proximal column did not react with anti- $\alpha 4$, but those in the uterine wall were $\alpha 4$ positive (Fig. $7 \mathrm{~B}$ ). These cells continued to react with this antibody after they penetrated the spiral arterioles (Fig. $7 \mathrm{D}$ ). The VCAM-1 and integrin $\alpha 4$ staining patterns were essentially the same as we observed in first trimester placental bed biopsy samples (18) and did not change in the third trimester (data not shown).

When the pregnancy was complicated by preeclampsia, staining for VCAM-1 was not detected on cytotrophoblasts in the decidua (Fig. $6 F$ ). The few cytotrophoblasts that reached the termini of spiral arterioles showed only weak antibody reactivity (Fig. $6 H$ ). However, integrin $\alpha 4$ expression on cytotrophoblasts in preeclamptic specimens was the same as in agematched, control tissue (Fig. 7, $F$ and $H$ ).

Finally, we examined cytotrophoblast staining for platelet endothelial adhesion molecule-1 (PECAM-1) in placental bed biopsies obtained at the same gestational age from control and preeclamptic patients. As its name suggests, PECAM-1 is present on platelets and on endothelium; it is also detected on certain leukocyte subsets. PECAM-1 can interact with itself, or with $\alpha \mathrm{V} \beta 3$ (26). In control tissue at 26 wk of gestation, endothelial cells present in anchoring and floating villi stained strongly for this adhesion molecule (Fig. $8 \mathrm{C}$ ). Cytotropho- blasts in villi did not stain for PECAM-1, but staining was detected on cytotrophoblasts within cell columns (data not shown). Cells involved in both interstitial (Fig. $8 D$ ) and endovascular invasion (Fig. $8 F$ ) stained particularly brightly with antibodies that recognized this adhesion molecule. Essentially the same staining pattern was observed in all three trimesters. In contrast, anti-PECAM-1 failed to stain preeclamptic cytotrophoblasts, both in the columns and in the decidua (Fig. $8 H$ ). However, maternal endothelial cells that lined the uterine vessels stained brightly (Fig. $8 J$ ).

\section{Discussion}

We became interested in studying the adhesion repertoire of cytotrophoblasts that participate in endovascular invasion when we noticed that these cells fail to stain with antibodies that recognize $\alpha 6 \beta 4$, an integrin receptor expressed by several epithelial stem cells, including cytotrophoblasts that are anchored to the villus basement membrane (11). However, cytotrophoblasts in columns upregulate expression of integrin $\alpha 1 \beta 1$ and endovascular cytotrophoblasts stain particularly brightly for this extracellular matrix receptor, which is widely expressed by embryonic blood vessels and activated leukocytes $(27,28)$. We also find that in preeclampsia, a disease in which endovascular invasion is abnormally shallow, cytotrophoblasts that invade the uterine wall stain for $\alpha 6 \beta 4$, but no $\alpha 1 \beta 1$ expression is detected (9). Together, these results suggested to us that in normal pregnancy, cytotrophoblasts differentiating along the invasive pathway change their adhesion phenotype from one that is characteristic of epithelial cells to one that is characteristic of vascular cells. This hypothesis is strongly supported by data presented in the accompanying paper (18). We also reasoned that in preeclampsia cytotrophoblasts might fail to properly execute this transformation. The latter hypothesis is strongly supported by data in this paper.

This dramatic switch in the cytotrophoblast adhesion molecule repertoire is likely to have many important functional consequences. Thus far, we have primarily focused on invasiveness. Our in vitro analyses of the role played by individual adhesion molecules suggest that in normal pregnancy, cytotrophoblast stem cells stain for adhesion receptors that inhibit invasion (e.g., E-cadherin [18] and $\alpha 6 \beta 4$ [12]). Within the column, expression of these molecules is downregulated in favor of receptors that promote invasion (e.g., $\alpha 1 \beta 1$ [12], $\alpha \mathrm{V} \beta 3$ and VE-cadherin [18]). Integrin $\alpha 5 \beta 1$, the fibronectin receptor, is an interesting exception. Expression of this receptor, which inhibits invasion in vitro (12), is first detected in the cell column, suggesting that differentiating/invading cytotrophoblasts still have adhesion mechanisms for counterbalancing invasiveness.

Taken together, the results presented here and in the accompanying paper (18) suggest that in preeclampsia, cytotrophoblasts start to differentiate along the invasive pathway but do not complete this process. Consequently, their ability to invade the uterus is likely to be impaired. With regard to their capacity to differentiate, cells in the anchoring villi and uterine wall fail to undergo the epithelial-to-vascular transformation in their adhesion molecule phenotype that occurs in normal pregnancy. They display staining for $\alpha 6 \beta 4, \alpha \mathrm{V} \beta 6$, and E-cadherin, adhesion molecules that are normally expressed by cytotrophoblast stem cells and/or cells in the proximal column region. At the same time they fail to switch on $\alpha \mathrm{V} \beta 3, \alpha 1 \beta 1$, VE-cadherin, VCAM-1, and PECAM-1, receptors that are normally expressed by the 

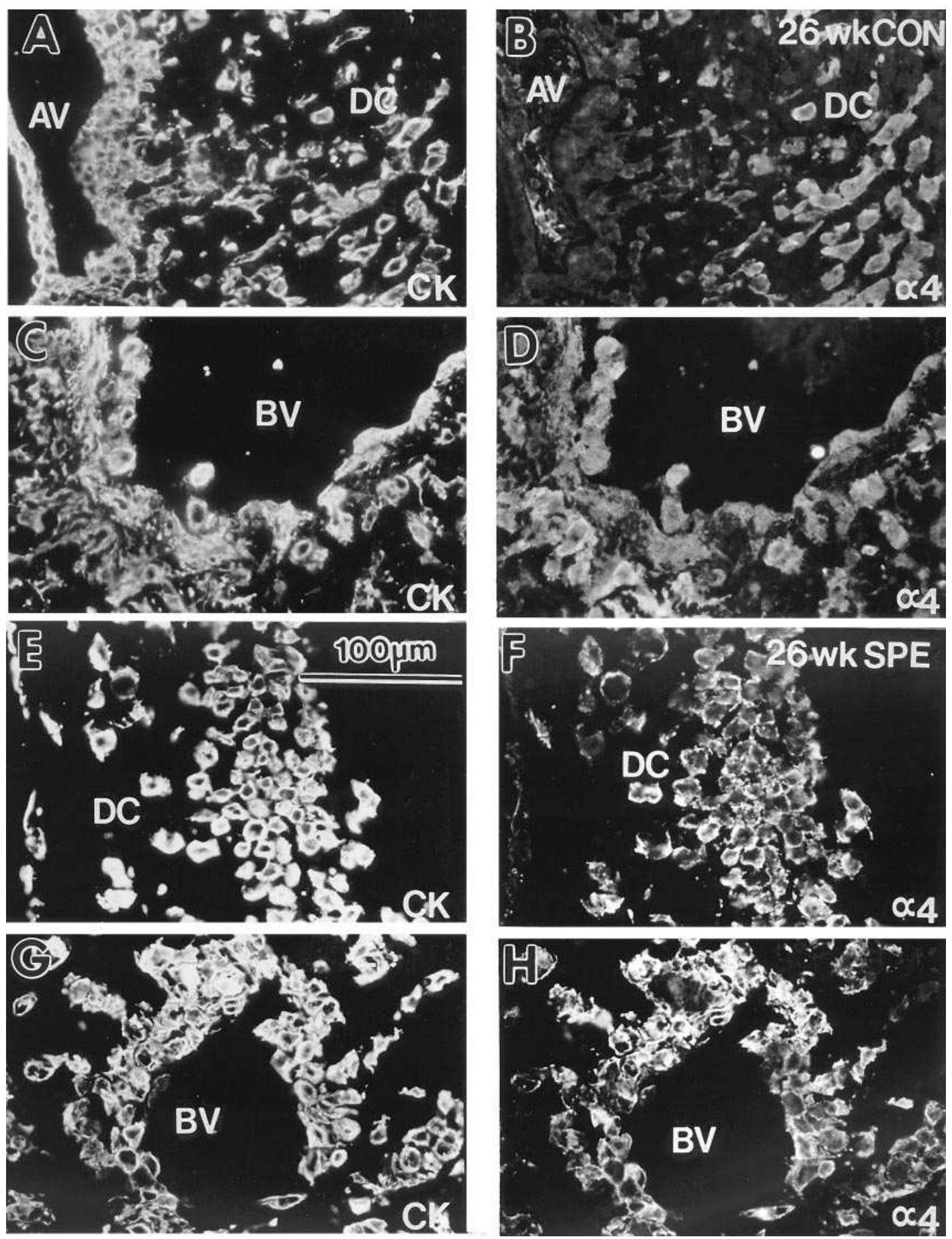

Figure 7. The integrin $\alpha 4$ was regulated normally in preeclampsia. Sections of age-matched 26-wk control (CON, $A-D)$ and severe preeclamptic ( $S P E, E-H)$ tissue double stained for CK $(A, C, E$, and $G)$ and $\alpha 4$ integrin $(B, D$, $F$, and $H) .(A, B)$ Control anchoring villus and placental bed. Staining for $\alpha 4$ was strong on stromal cells in the villus core but weak or not detected on villus CTB. Staining intensity increased on decidual CTB. $(C, D)$ Maternal blood vessel lined with CTB. Staining for $\alpha 4$ was detected on both CTB associated with the blood vessel and those in the surrounding uterine interstitium. In preeclamptic tissue, $\alpha 4$ integrin was detected on CTB within decidua $(E, F)$ and associated with maternal blood vessels $(G, H)$.

most differentiated and invasive population of cytotrophoblasts. With regard to invasiveness, our in vitro analyses suggest that the particular combination of adhesion molecules they do express (e.g., $\alpha 6 \beta 4, \alpha 5 \beta 1$, and E-cadherin) inhibits cytotrophoblast invasion $(12,18)$. This observation is consistent with the shallow placentation often associated with preeclampsia.

We have also been interested in whether this defect is permanent, or can be reversed by placing preeclamptic cytotrophoblasts in culture. Because of difficulty in obtaining control and experimental samples that are matched for gestation age, this study has taken several years. To date we have informa- tion on preeclampsia's effects on cytotrophoblast $\alpha 1 \beta 1$ expression and invasiveness. These data show that by the time the symptoms necessitate delivery, the negative effects of preeclampsia on cytotrophoblast differentiation/invasion cannot be reversed by placing the cells in culture (Lim, K.H., Y. Zhou, M. Janatpour, M. McMaster, K. Bass, S.-H. Chun, and S.J. Fisher, manuscript submitted for publication). Currently, we are investigating whether this is also true of cytotrophoblast expression of antigens that mark the epithelial-to-vascular transition.

The extensive mimicry by normal cytotrophoblasts of the 

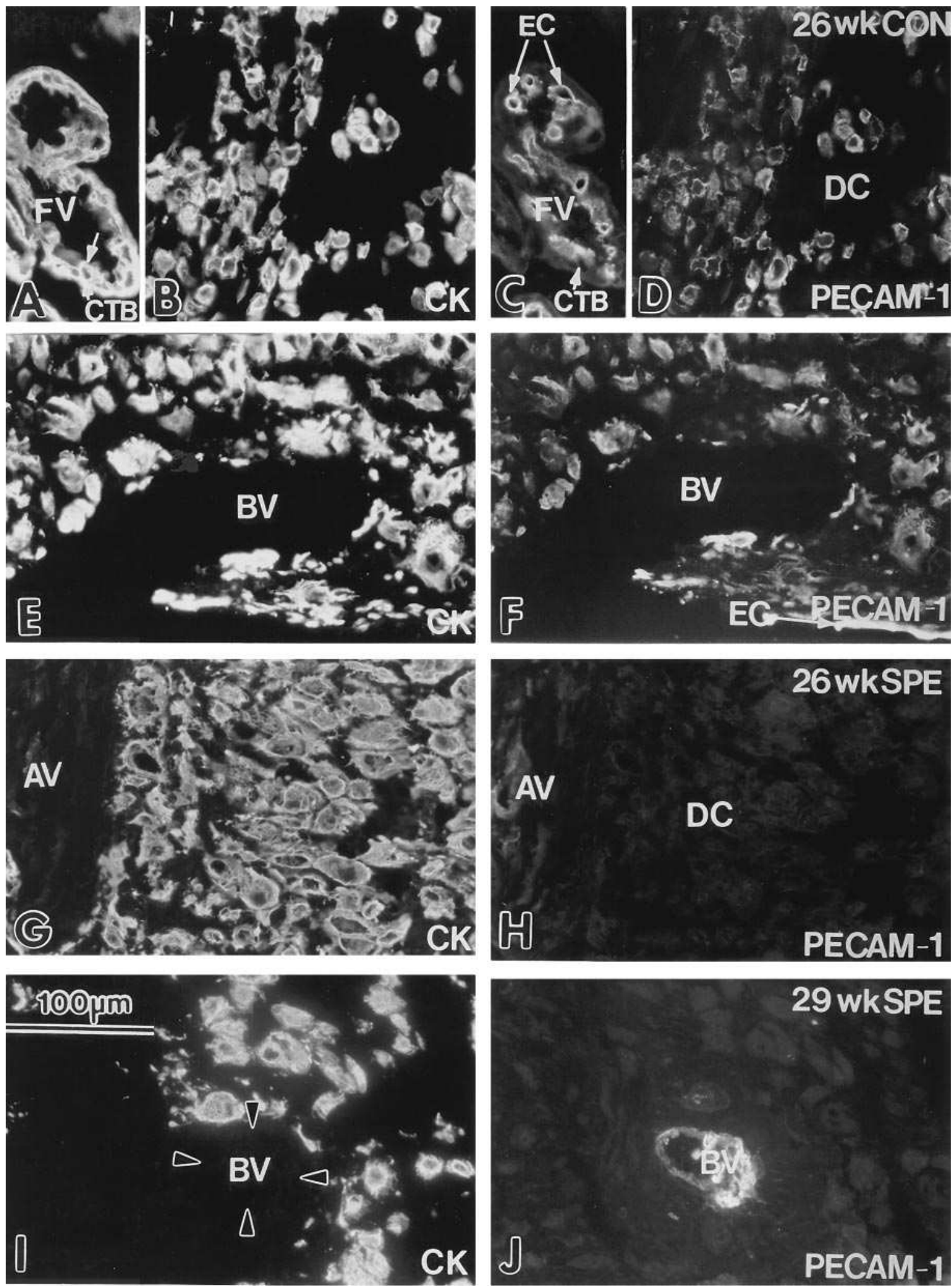

Figure 8. Staining for PECAM-1 was not detected on preeclamptic CTB. Sections of age-matched 26-wk control $(C O N, A-F)$ and severe preeclamptic $(S P E, G-J)$ tissue double stained for CK $(A, B, E, G$, and $I)$ and PECAM-1 $(C, D, F, H$, and $J)$. $(A, C)$ Sections of control chorionic floating villus $(F V)$ showed intense staining for PECAM-1 on fetal blood vessels (arrows in $C$ ), but not on CTB (arrows in $A$ ). PECAM-1 staining was detected on uterine wall CTB $(B, D)$ and on both CTB (CK-positive) and maternal endothelial cells $(E C)$ associated with a maternal blood vessel $(B V)(E, F)$. In SPE, PECAM-1 staining was detected on endothelium of fetal blood vessels in anchoring villi $(A V)$ (data not shown) and on the unmodified maternal vessels in the uterine wall $(J)$, but CTB did not stain in any location. 
adhesion receptor phenotype of endothelial cells is likely to play an equally important role in the process whereby these cells form vascular connections with the uterine vessels. Ultimately these connections are so extensive that the spiral arterioles become hybrid structures in which fetal cytotrophoblasts replace the maternal endothelium and much of the highly muscular tunica media. As a result, the diameter of the spiral arterioles increases dramatically, allowing blood flow to the placenta to keep pace with fetal growth. Circumstantial evidence suggests that several of the adhesion molecules whose expression we studied could play an important role in forming these novel vascular connections. For example, in the mouse, targeted disruption of either VCAM- 1 or $\alpha 4$ expression results in failure of chorioallantoic fusion $(29,30)$. It is also very interesting to find that cytotrophoblasts are the only cells, other than the endothelium, that express VE-cadherin. In addition, VEcadherin and PECAM-1 are the first adhesion receptors expressed by differentiating endothelial cells during early development (31). $\alpha \mathrm{V} \beta 3$ expression is upregulated on endothelial cells during angiogenesis by soluble factors that regulate this process (23). Thus, adhesion receptors that are upregulated as normal cytotrophoblasts differentiate/invade play vital roles in differentiation and expansion of the vasculature. This raises the interesting possibility that the failure of preeclamptic cytotrophoblasts to express these adhesion molecules also impairs their ability to form vascular connections with the uterine vessel. This failure ultimately limits the supply of maternal blood to the placenta and fetus, an effect thought to be closely linked to the pathophysiology of the disease (10).

We are also interested in the possibility that the failure of preeclamptic cytotrophoblasts to express endothelial-type adhesion molecules has important consequences on other types of cell-cell interactions that involve uterine vessels. For example, the decidua contains large numbers of unusual subsets of maternal immune cells (32). Leukocyte extravasation at other sites requires a complex set of interactions mediated by selectins, Ig superfamily members and integrins $(33,34)$. Whether leukocyte extravasation into the uterus is influenced by endovascular cytotrophoblasts that colonize the superficial portions of maternal arterioles has not been investigated. However, it is interesting to note that preeclamptic cytotrophoblasts stain very weakly for VCAM-1, the endothelial receptor for leukocyte $\alpha 4 \beta 1$. Thus, it is possible that the way maternal leukocytes interact with these uterine vessels also changes. As a result, the phenotype of decidual leukocytes might be different in preeclampsia, a possibility we are currently examining. Preeclamptic cytotrophoblasts also fail to express PECAM-1, raising the additional possibility that they have faulty interactions with platelets, a particularly intriguing possibility because platelet activation is a frequent complication of preeclampsia (35).

Finally, we hypothesize that the failure of preeclamptic cytotrophoblasts to make a transition to a vascular cell adhesion phenotype might be part of a broader spectrum defect in which the cells fail to function properly as endothelium. Such a failure would no doubt have important effects on the maintenance of vascular integrity at the maternal-fetal interface. Clearly, in preeclampsia undifferentiated cytotrophoblasts that fail to mimic the adhesion phenotype of endothelial cells are present in the termini of maternal spiral arterioles. Whether this phenomenon affects the phenotype of maternal endothelium in deeper segments of the same vessels, and/or is linked to the maternal endothelial pathology that is a hallmark of this disease, is an interesting question that remains to be investigated.

\section{Acknowledgments}

The authors thank Mr. Chanh Dinh for assistance in the preparation of figures and Evangeline Leash for excellent editorial assistance.

This work was supported by HD 30367 and HD 22210, and by a Postdoctoral Fellowship (to Y. Zhou) from The Rockefeller Foundation.

\section{References}

1. Roberts, J.M., R.N. Taylor, S. Friedman, and A. Goldfien. 1990. New developments in preeclampsia. In Fetal Medical Review. W. Dunlop, editor. Edward Arnold, London. 125-141.

2. Roberts, J.M., R.N. Taylor, T.J. Musci, G.M. Rodgers, C.A. Hubel, and M.K. McLaughlin. 1989. Preeclampsia: an endothelial cell disorder. Am. J. Obstet. Gynecol. 161:1200-1204.

3. Berg, C.G., H.K. Atrash, L.M. Koonin, and M. Tucker. 1996. Pregnancyrelated mortality in the United States. Obstet. Gynecol. 88:161-167.

4. Redman, C.W. 1990. Platelets and the beginnings of preeclampsia. $N$. Engl. J. Med. 323:478-480.

5. Chun, D., C. Braga, C. Chow, and L. Lok. 1964. Clinical observations on some aspects of hydatidiform moles. J. Obstet. Gynecol. Br. Commonw. 71:180-84.

6. Brosens, I.A., W.B. Robertson, and H.G. Dixon. 1972. The role of the spiral arteries in the pathogenesis of preeclampsia. Obstet. Gynecol. Annu. 1: 177-191.

7. Gerretsen, G., H.J. Huisjes, and J.D. Elema. 1981. Morphological changes of the spiral arteries in the placental bed in relation to pre-eclampsia and fetal growth retardation. Br. J. Obstet. Gynaecol. 88:876-881.

8. Khong, T.Y., W.F. De, W.B. Robertson, and I. Brosens. 1986. Inadequate maternal vascular response to placentation in pregnancies complicated by preeclampsia and by small-for-gestational age infants. Br. J. Obstet. Gynaecol. 93: 1049-1059.

9. Zhou, Y., C.H. Damsky, K. Chiu, J.M. Roberts, and S.J. Fisher. 1993 Preeclampsia is associated with abnormal expression of adhesion molecules by invasive cytotrophoblasts. J. Clin. Invest. 91:950-960.

10. Harrington, K., and S. Campbell. 1992. Doppler ultrasound in prenatal prediction and diagnosis. Curr. Opin. Obstet. Gynecol. 4:264-272.

11. Damsky, C.H., M.L. Fitzgerald, and S.J. Fisher. 1992. Distribution patterns of extracellular matrix components and adhesion receptors are intricately modulated during first trimester cytotrophoblast differentiation along the invasive pathway in vivo. J. Clin. Invest. 89:210-222.

12. Damsky, C.H., C. Librach, K.H. Lim, M.L. Fitzgerald, M.T. McMaster, M. Janatpour, Y. Zhou, S.K. Logan, and S.J. Fisher. 1994. Integrin switching regulates normal trophoblast invasion. Development (Camb.). 120:3057-3066.

13. Librach, C.L., Z. Werb, M.L. Fitzgerald, K. Chiu, N.M. Corwin, R.A. Esteves, D. Grobelny, R. Galardy, C.H. Damsky, and S.J. Fisher. 1991. 92-kD type IV collagenase mediates invasion of human cytotrophoblasts. J. Cell Biol. 113:437-449.

14. Kovats, S., E.K. Main, C. Librach, M. Stubblebine, S.J. Fisher, and R. DeMars. 1990. A class I antigen, HLA-G, expressed in human trophoblasts. Science (Wash. DC). 248:220-223.

15. McMaster, M.T., C. Librach, Y. Zhou, K.H. Lim, M.J. Janatpour, R DeMars, S. Kovats, C. Damsky, and S.J. Fisher. 1995. Human placental HLA-G expression is restricted to differentiated cytotrophoblasts. J. Immunol. 153: 3771-3778.

16. Redline, R.W., and P. Patterson. 1995. Pre-eclampsia is associated with an excess of proliferative immature intermediate trophoblast. Hum. Pathol. 26: 594-600.

17. Genbacev, O., R. Joslin, C.H. Damsky, B.M. Polliotti, and S.J. Fisher. 1996. Hypoxia alters early gestation human cytotrophoblast differentiation/invasion in vitro and models the placental defects that occur in preeclampsia. $J$. Clin. Invest. 97:540-550.

18. Zhou, Y., S.J. Fisher, M. Janatpour, O. Genbacev, E. Dejena, M. Wheelock, and C.H. Damsky. 1997. Human cytotrophoblasts adopt a vascular phenotype as they differentiate: a strategy for successful endovascular invasion? $J$. Clin. Invest. 99:2139-2151.

19. Chesley, L. 1985. Diagnostic criteria for preeclampsia. Obstet. Gynecol. 65:423-425.

20. 1996. Hypertensive diseases in pregnancy. American College of Obstetricians and Gynecologists Technical Bulletin. 219.

21. Sibai, B.M. 1990. The HELLP syndrome (hemolysis, elevated liver enzymes, and low platelets): much ado about nothing? Am. J. Obstet. Gynecol. 162:311-316.

22. Breuss, J.M., J. Gallo, H.M. DeLisser, I.V. Klimanskaya, H.G. Folkesson, J.F. Pittet, S.L. Nishimura, K. Aldape, D.V. Landers, W. Carpenter, et al. 
1995. Expression of the beta 6 integrin subunit in development, neoplasia and tissue repair suggests a role in epithelial remodeling. J. Cell Sci. 108:2241-2251.

23. Brooks, P.C., R.A. Clark, and D.A. Cheresh. 1994. Requirement of vascular integrin alpha v beta 3 for angiogenesis. Science (Wash. DC). 264:569-571.

24. Birchmeier, W. 1995. E-cadherin as a tumor (invasion) suppresser gene. Bioessays. 17:97-99.

25. Carlos, T.M., and J.M. Harlan. 1994. Leukocyte-endothelial adhesion molecules. Blood. 84:2068-2101.

26. Piali, L., P. Hammel, C. Uherek, F. Bachmann, R.H. Gisler, D. Dunon, and B.A. Imhof. 1995. CD31/PECAM-1 is a ligand for alpha v beta 3 integrin involved in adhesion of leukocytes to endothelium. J. Cell Biol. 130:451-460.

27. Hemler, M.E., J.G. Jacobson, M.B. Brenner, D. Mann, and J.L. Strominger. 1985. VLA-1: a T cell surface antigen which defines a novel late stage of human T cell activation. Eur. J. Immunol. 15:502-508.

28. Belkin, V.M., A.M. Belkin, and V.E. Koteliansky. 1990. Human smooth muscle VLA-1 integrin: purification, substrate specificity, localization in aorta, and expression during development. J. Cell Biol. 111:2159-2170.

29. Gurtner, G.C., V. Davis, H. Li, M.J. McCoy, A. Sharpe, and M.I. Cybul- sky. 1995. Targeted disruption of the murine VCAM1 gene: essential role of VCAM-1 in chorioallantoic fusion and placentation. Genes Dev. 9:1-14.

30. Yang, J.T., H. Rayburn, and R.O. Hynes. 1995. Cell adhesion events mediated by alpha 4 integrins are essential in placental and cardiac development. Development (Camb.). 121:549-560.

31. Risau, W., and I. Flamme. 1995. Vasculogenesis. Ann. Rev. Cell Dev. Biol. 11:73-91.

32. Starkey, P.M., I.L. Sargent, and C.W. Redman. 1988. Cell populations in human early pregnancy decidua: characterization and isolation of large granular lymphocytes by flow cytometry. Immunology. 65:129-134.

33. Rosen, S.D. 1993. Cell surface lectins in the immune system. Semin. Immunol. 5:237-47.

34. Girard, J.P., and T.A. Springer. 1995. High endothelial venules (HEVs): specialized endothelium for lymphocyte migration. Immunol. Today. 16:449457.

35. Janes, S.L., P.M. Kyle, C. Redman, and A.H. Goodall. 1995. Flow cytometric detection of activated platelets in pregnant women prior to the development of pre-eclampsia. Thromb. Haemostasis. 74:1059-1063. 